\title{
Local Fractional Laplace Variational Iteration Method for Fractal Vehicular Traffic Flow
}

\author{
Yang Li, ${ }^{1}$ Long-Fei Wang, ${ }^{1}$ Sheng-Da Zeng, ${ }^{2}$ and Yang Zhao ${ }^{3}$ \\ ${ }^{1}$ School of Highway, Chang'an University, Xian 710064, China \\ ${ }^{2}$ School of Science, Guangxi University for Nationalities, Nanning 530006, China \\ ${ }^{3}$ College of Instrumentation \& Electrical Engineering, Jilin University, Changchun 130061, China \\ Correspondence should be addressed to Yang Zhao; zhaoyang19781023@gmail.com
}

Received 10 May 2014; Accepted 20 May 2014; Published 29 June 2014

Academic Editor: Xiao-Jun Yang

Copyright (c) 2014 Yang Li et al. This is an open access article distributed under the Creative Commons Attribution License, which permits unrestricted use, distribution, and reproduction in any medium, provided the original work is properly cited.

We discuss the line partial differential equations arising in fractal vehicular traffic flow. The nondifferentiable approximate solutions are obtained by using the local fractional Laplace variational iteration method, which is the coupling method of local fractional variational iteration method and Laplace transform. The obtained results show the efficiency and accuracy of implements of the present method.

\section{Introduction}

Fractional differential equations with arbitrary orders were applied to model the real-world problems for science and engineering. Many researchers present their applications with solid mechanics, heat transfer, fluid mechanics, transport process, water motion, and quantum mechanics. For example, Tarasov studied the wave equation for fractal solid string based on the fractional calculus [1]. Momani and Odibat presented the linear, nonlinear, and fractional partial differential equations arising in fluid mechanics $[2,3]$. Povstenko suggested the fractional heat conduction equation [4], and Vázquez gave the second law of thermodynamics with fractional derivative [5]. Lutz considered the fractional transport equation for Levy stable processes [6], and Kadem et al. discussed its solution using the spectral method [7]. Laskin presented the fractional Schrodinger equation [8], and Muslih et al. suggested its solution [9]. There are many methods for finding the solutions to the fractional differentiable equations [10]. For example, Jafari and Seifi used the homotopy analysis method to deal with linear and nonlinear fractional diffusion-wave equations [11]. Jafari et al. applied the fractional subequation method to solve the CahnHilliard and Klein-Gordon equations [12]. Hristov suggested the heat-balance integral method for fractional heat diffusion and transient flow $[13,14]$. Bhrawy and Alghamdi proposed the shifted Jacobi-Gauss-Lobatto collocation method to find the solution for nonlinear fractional Langevin equation with two variables [15] and the shifted Legendre spectral method for solving the fractional-order multipoint boundary value problems [16]. Bhrawy and Baleanu considered the spectral Legendre-Gauss-Lobatto collocation method to solve the space-fractional advection diffusion equations [17]. Atangana and Baleanu presented the two difference methods to solve the fractional parabolic equations [18].

Recently, the local fractional calculus is proposed and developed to describe the fractal problems in various fields, such as physics [19-21], applied mathematics [22, 23], signal processing [24-27], fluid mechanics [28], quantum mechanics [29], fractal forest gap [30], vehicular traffic flow [31], and silk cocoon hierarchy [32]. The linear differential equation arising in fractal vehicular traffic flow was suggested in [31]. The local fractional Laplace variational iteration method was suggested in [23] and developed in [33]. In this paper, we use the local fractional Laplace variational iteration method to solve the linear differential equation arising in fractal vehicular traffic flow. The structure of the paper is suggested as follows. In Section 2, the basic theory of local fractional calculus and local fractional Laplace transform are introduced. Section 3 gives the local fractional Laplace variational 
iteration method. In Section 4, the nondifferentiable solutions for line partial differential equations arising in fractal vehicular traffic flow are presented. Finally, the conclusions are considered in Section 5.

\section{The Lighthill-Whitham-Richards Model on a Finite Length Highway}

In this section, we present the Lighthill-Whitham-Richards model on a finite length highway, the conceptions of local fractional derivative and integral, and the local fractional Laplace transform.

The Lighthill-Whitham-Richards model on a finite length highway reads as follows [31]:

$$
\frac{\partial^{\alpha} \varphi(x, t)}{\partial t^{\alpha}}+\mu \frac{\partial^{\alpha} \varphi(x, t)}{\partial x^{\alpha}}=0
$$

where the initial and boundary conditions are presented as follows:

$$
\begin{gathered}
\varphi(x, 0)=\varphi_{0}(x), \\
\varphi(0, t)=\omega(t),
\end{gathered}
$$

with

$$
\begin{gathered}
\left|\varphi_{0}(x)-\varphi_{0}\left(x_{0}\right)\right|<\varepsilon^{\alpha}, \\
\left|\omega(t)-\omega\left(t_{0}\right)\right|<\kappa^{\alpha},
\end{gathered}
$$

for $\left|x-x_{0}\right|<\delta,\left|t-t_{0}\right|<\tau$ for $\varepsilon, \kappa, \delta, \tau>0,0<\alpha<1$, and the local fractional partial derivative of $f(x)$ of order $\alpha$ is given as [19]

$$
\frac{d^{\alpha} f\left(x_{0}\right)}{d x^{\alpha}}=\frac{\Delta^{\alpha}\left(f(x)-f\left(x_{0}\right)\right)}{\left(x-x_{0}\right)^{\alpha}},
$$

with

$$
\Delta^{\alpha}\left(f(x)-f\left(x_{0}\right)\right) \cong \Gamma(1+\alpha)\left[f(x)-f\left(x_{0}\right)\right] .
$$

This is the line partial differential equation arising in fractal vehicular traffic flow.

The local fractional derivative of $f(x)$ of order $\alpha$ is expressed as $[19,20]$

$$
\frac{d^{\alpha} f\left(x_{0}\right)}{d x^{\alpha}}=\frac{\Delta^{\alpha}\left(f(x)-f\left(x_{0}\right)\right)}{\left(x-x_{0}\right)^{\alpha}},
$$

where

$$
\Delta^{\alpha}\left(f(x)-f\left(x_{0}\right)\right) \cong \Gamma(1+\alpha)\left[f(x)-f\left(x_{0}\right)\right] .
$$

The local fractional integral of $f(x)$ of order $\alpha$ in the interval $[a, b]$ is defined through $[19,21,22]$

$$
\begin{aligned}
a_{b}^{(\alpha)} f(x) & =\frac{1}{\Gamma(1+\alpha)} \int_{a}^{b} f(t)(d t)^{\alpha} \\
& =\frac{1}{\Gamma(1+\alpha)} \lim _{\Delta t \rightarrow 0} \sum_{j=0}^{j=N-1} f\left(t_{j}\right)\left(\Delta t_{j}\right)^{\alpha},
\end{aligned}
$$

where the partitions of the interval $[a, b]$ are $\left(t_{j}, t_{j+1}\right)$, with $\Delta t_{j}=t_{j+1}-t_{j}, t_{0}=a, t_{N}=b$, and $\Delta t=\max \left\{\Delta t_{0}, \Delta t_{1}, \Delta t_{j}\right.$, $\ldots\}, j=0, \ldots, N-1$. $33-35$

The local fractional Laplace transform is given as $[23,26$,

$$
\begin{aligned}
& \tilde{L}_{\alpha}\{f(x)\}= f_{s}^{\widetilde{L}, \alpha}(s) \\
&=\frac{1}{\Gamma(1+\alpha)} \int_{0}^{\infty} E_{\alpha}\left(-s^{\alpha} x^{\alpha}\right) f(x)(d x)^{\alpha}, \\
& \quad 0<\alpha \leq 1,
\end{aligned}
$$

where $f(x)$ is local fractional continuous and $s^{\alpha}=\beta^{\alpha}+i^{\alpha} \infty^{\alpha}$.

The inverse formula of local fractional Laplace transform is suggested as $[23,26,33-35]$

$$
\begin{aligned}
f(x) & =\widetilde{L}_{\alpha}^{-1}\left\{f_{s}^{L, \alpha}(s)\right\} \\
& =\frac{1}{(2 \pi)^{\alpha}} \int_{\beta-i \infty}^{\beta+i \infty} E_{\alpha}\left(s^{\alpha} x^{\alpha}\right) f_{s}^{\widetilde{L}, \alpha}(s)(d s)^{\alpha},
\end{aligned}
$$

where $s^{\alpha}=\beta^{\alpha}+i^{\alpha} \infty^{\alpha}$ and $\operatorname{Re}\left(s^{\alpha}\right)=\beta^{\alpha}$.

The local fractional convolution of two functions is presented as $[23,33]$

$$
f_{1}(x) * f_{2}(x)=\frac{1}{\Gamma(1+\alpha)} \int_{-\infty}^{\infty} f_{1}(t) f_{2}(x-t)(d t)^{\alpha} .
$$

The properties for local fractional Laplace transform are listed as follows [23, 26, 33-35]:

$$
\begin{gathered}
\widetilde{L}_{\alpha}\{a f(x)+b g(x)\}=a \widetilde{L}_{\alpha}\{f(x)\}+b \widetilde{L}_{\alpha}\{g(x)\} \\
\widetilde{L}_{\alpha}\left\{f^{(n \alpha)}(x)\right\}=s^{n \alpha} \widetilde{L}_{\alpha}\{f(x)\}-\sum_{k=1}^{n} s^{(k-1) \alpha} f^{(n-k) \alpha}(0), \\
F_{\alpha}\left\{f_{1}(x) * f_{2}(x)\right\}=f_{\omega, 1}^{F, \alpha}(\omega) f_{\omega, 2}^{F, \alpha}(\omega), \\
\widetilde{L}_{\alpha}\left\{\sin _{\alpha}\left(c^{\alpha} x^{\alpha}\right)\right\}=\frac{c^{\alpha}}{s^{2 \alpha}+c^{2 \alpha}} \\
\widetilde{L}_{\alpha}\left\{\cos _{\alpha}\left(c^{\alpha} x^{\alpha}\right)\right\}=\frac{s^{\alpha}}{s^{2 \alpha}+c^{2 \alpha}} \\
\widetilde{L}_{\alpha}\left\{x^{k \alpha}\right\}=\frac{\Gamma(1+k \alpha)}{s^{(k+1) \alpha}} .
\end{gathered}
$$

\section{Local Fractional Laplace Variational Iteration Method}

In this section, we introduce the local fractional Laplace variational iteration method. Let us consider the following local fractional differential operator:

$$
L_{\alpha} u-R_{\alpha} u=0
$$

where the linear local fractional differential operator is $L_{\alpha}=$ $\left(d^{\alpha} / d s^{\alpha}\right)$ and $u(x)$ is a nondifferential function. 
According to the local fractional Laplace variational iteration method [23, 33], the local fractional functional formula is presented as follows:

$$
u_{n+1}(x)=u_{n}(x)+{ }_{0} I_{x}^{(\alpha)}\left\{\frac{\lambda(x-t)^{\alpha}}{\Gamma(1+\alpha)}\left[L_{\alpha} u_{n}(t)-R_{\alpha} u_{n}\right]\right\} .
$$

Applying the local fractional Laplace transform gives the following:

$$
\begin{aligned}
\widetilde{L}_{\alpha}\left\{u_{n+1}(x)\right\}= & \widetilde{L}_{\alpha}\left\{u_{n}(x)\right\} \\
& +\widetilde{L}_{\alpha}\left\{\frac{\lambda(x)^{\alpha}}{\Gamma(1+\alpha)}\right\} \widetilde{L}_{\alpha}\left\{L_{\alpha} u_{n}(x)-R_{\alpha} u_{n}(x)\right\} .
\end{aligned}
$$

Taking the local fractional variation of (15), we obtain

$$
\begin{aligned}
\delta^{\alpha}\left\{\widetilde{L}_{\alpha}\left\{u_{n+1}(x)\right\}\right\} \\
=\delta^{\alpha}\left\{\widetilde{L}_{\alpha}\left\{u_{n}(x)\right\}\right\} \\
\quad+\delta^{\alpha}\left\{\widetilde{L}_{\alpha}\left\{\frac{\lambda(x)^{\alpha}}{\Gamma(1+\alpha)}\right\} \widetilde{L}_{\alpha}\left\{L_{\alpha} u_{n}(x)-R_{\alpha} u_{n}(x)\right\}\right\} .
\end{aligned}
$$

From (16), we have

$$
\begin{aligned}
& \delta^{\alpha}\left\{\widetilde{L}_{\alpha}\left\{u_{n+1}(x)\right\}\right\} \\
&=\delta^{\alpha}\left\{\widetilde{L}_{\alpha}\left\{u_{n}(x)\right\}\right\} \\
&+\widetilde{L}_{\alpha}\left\{\frac{\lambda(x)^{\alpha}}{\Gamma(1+\alpha)}\right\}\left\{\delta^{\alpha}\left\{\widetilde{L}_{\alpha}\left\{L_{\alpha} u_{n}(x)\right\}\right\}\right\}=0
\end{aligned}
$$

such that

$$
1+\widetilde{L}_{\alpha}\left\{\frac{\lambda(x)^{\alpha}}{\Gamma(1+\alpha)}\right\} s^{\alpha}=0
$$

where

$$
\begin{aligned}
\delta^{\alpha}\left\{\widetilde{L}_{\alpha}\left\{L_{\alpha} u_{n}(x)\right\}\right\} & =\delta^{\alpha}\left\{s^{\alpha} \widetilde{L}_{\alpha}\left\{u_{n}(x)\right\}-u_{n}(0)\right\} \\
& =s^{\alpha} \delta^{\alpha} \widetilde{L}_{\alpha}\left\{u_{n}(x)\right\} .
\end{aligned}
$$

Therefore, we get

$$
\widetilde{L}_{\alpha}\left\{\frac{\lambda(x)^{\alpha}}{\Gamma(1+\alpha)}\right\}=-\frac{1}{s^{\alpha}} .
$$

Using formula (20), we arrive at new iteration algorithm as follows:

$$
\begin{aligned}
\widetilde{L}_{\alpha}\left\{u_{n+1}(x)\right\}= & \widetilde{L}_{\alpha}\left\{u_{n}(x)\right\} \\
& -\frac{1}{s^{\alpha}} \widetilde{L}_{\alpha}\left\{\left(L_{\alpha} u_{n}(x)-R_{\alpha} u_{n}(x)\right)\right\},
\end{aligned}
$$

where the initial value reads as

$$
\widetilde{L}_{\alpha}\left\{u_{0}(x)\right\}=u(0) .
$$

Thus, the local fractional series solution of (13) is

$$
\widetilde{L}_{\alpha}\{u\}=\lim _{n \rightarrow \infty} \widetilde{L}_{\alpha}\left\{u_{n}\right\}
$$

which leads to

$$
u=\lim _{n \rightarrow \infty} \widetilde{L}_{\alpha}^{-1}\left\{\widetilde{L}_{\alpha} u_{n}\right\}
$$

\section{The Nondifferentiable Solutions for Line Partial Differential Equations Arising in Fractal Vehicular Traffic Flow}

In this section, we present the boundary value problems for line partial differential equations arising in fractal vehicular traffic flow.

Example 1. The initial and boundary conditions for line partial differential equations arising in fractal vehicular traffic flow read as follows:

$$
\begin{gathered}
\varphi(x, 0)=E_{\alpha}\left(x^{\alpha}\right), \\
\varphi(0, t)=0 .
\end{gathered}
$$

In view of (21), we have

$$
\begin{aligned}
\widetilde{L}_{\alpha} & \left\{\varphi_{n+1}(x, t)\right\} \\
= & \widetilde{L}_{\alpha}\left\{\varphi_{n}(x, t)\right\}-\frac{1}{s^{\alpha}} \widetilde{L}_{\alpha}\left\{\frac{\partial^{\alpha} \varphi_{n}(x, t)}{\partial t^{\alpha}}+\mu \frac{\partial^{\alpha} \varphi_{n}(x, t)}{\partial x^{\alpha}}\right\} \\
= & \varphi_{n}(x, s)-\frac{1}{s^{\alpha}}\left\{s^{\alpha} \varphi_{n}(x, s)-\varphi_{n}(x, 0)+\mu \frac{\partial^{\alpha} \varphi_{n}(x, s)}{\partial x^{\alpha}}\right\} \\
& =\frac{1}{s^{\alpha}} \varphi_{n}(x, 0)-\frac{\mu}{s^{\alpha}} \frac{\partial^{\alpha} \varphi_{n}(s, t)}{\partial x^{\alpha}},
\end{aligned}
$$

where the initial value is

$$
\widetilde{L}_{\alpha}\left\{\varphi_{0}(x, s)\right\}=\varphi_{0}(x, s)=\widetilde{L}_{\alpha}\left\{E_{\alpha}\left(x^{\alpha}\right)\right\}=\frac{E_{\alpha}\left(x^{\alpha}\right)}{s^{\alpha}} .
$$

Making use of (26) and (27), we have the first approximation

$$
\begin{aligned}
\widetilde{L}_{\alpha} & \left\{\varphi_{1}(x, t)\right\} \\
= & \widetilde{L}_{\alpha}\left\{\varphi_{0}(x, t)\right\}-\frac{1}{s^{\alpha}} \widetilde{L}_{\alpha}\left\{\frac{\partial^{\alpha} \varphi_{0}(x, t)}{\partial t^{\alpha}}+\mu \frac{\partial^{\alpha} \varphi_{0}(x, t)}{\partial x^{\alpha}}\right\} \\
& =\varphi_{0}(x, s)-\frac{1}{s^{\alpha}}\left\{s^{\alpha} \varphi_{0}(x, s)-\varphi_{0}(x, 0)+\mu \frac{\partial^{\alpha} \varphi_{0}(x, s)}{\partial x^{\alpha}}\right\} \\
& =\frac{1}{s^{\alpha}} \varphi_{0}(x, 0)-\frac{\mu}{s^{\alpha}} \frac{\partial^{\alpha} \varphi_{0}(s, t)}{\partial x^{\alpha}} \\
& =\frac{E_{\alpha}\left(x^{\alpha}\right)}{s^{\alpha}}-\frac{\mu E_{\alpha}\left(x^{\alpha}\right)}{s^{2 \alpha}} .
\end{aligned}
$$


In view of (26) and (28), we arrive at the second approximation

$$
\begin{aligned}
\widetilde{L}_{\alpha} & \left\{\varphi_{2}(x, t)\right\} \\
& =\widetilde{L}_{\alpha}\left\{\varphi_{1}(x, t)\right\}-\frac{1}{s^{\alpha}} \widetilde{L}_{\alpha}\left\{\frac{\partial^{\alpha} \varphi_{1}(x, t)}{\partial t^{\alpha}}+\mu \frac{\partial^{\alpha} \varphi_{1}(x, t)}{\partial x^{\alpha}}\right\} \\
& =\varphi_{1}(x, s)-\frac{1}{s^{\alpha}}\left\{s^{\alpha} \varphi_{1}(x, s)-\varphi_{1}(x, 0)+\mu \frac{\partial^{\alpha} \varphi_{1}(x, s)}{\partial x^{\alpha}}\right\} \\
& =\frac{1}{s^{\alpha}} \varphi_{1}(x, 0)-\frac{\mu}{s^{\alpha}} \frac{\partial^{\alpha} \varphi_{1}(s, t)}{\partial x^{\alpha}} \\
& =\frac{E_{\alpha}\left(x^{\alpha}\right)}{s^{\alpha}}-\frac{\mu}{s^{\alpha}}\left(\frac{E_{\alpha}\left(x^{\alpha}\right)}{s^{\alpha}}-\frac{\mu E_{\alpha}\left(x^{\alpha}\right)}{s^{2 \alpha}}\right) \\
& =\frac{E_{\alpha}\left(x^{\alpha}\right)}{s^{\alpha}}-\frac{\mu E_{\alpha}\left(x^{\alpha}\right)}{s^{2 \alpha}}+\frac{\mu^{2} E_{\alpha}\left(x^{\alpha}\right)}{s^{3 \alpha}} .
\end{aligned}
$$

From (26) and (29), the third approximation is

$$
\begin{aligned}
\widetilde{L}_{\alpha} & \left\{\varphi_{3}(x, t)\right\} \\
& =\widetilde{L}_{\alpha}\left\{\varphi_{2}(x, t)\right\}-\frac{1}{s^{\alpha}} \widetilde{L}_{\alpha}\left\{\frac{\partial^{\alpha} \varphi_{2}(x, t)}{\partial t^{\alpha}}+\mu \frac{\partial^{\alpha} \varphi_{2}(x, t)}{\partial x^{\alpha}}\right\} \\
& =\varphi_{2}(x, s)-\frac{1}{s^{\alpha}}\left\{s^{\alpha} \varphi_{2}(x, s)-\varphi_{2}(x, 0)+\mu \frac{\partial^{\alpha} \varphi_{2}(x, s)}{\partial x^{\alpha}}\right\} \\
& =\frac{1}{s^{\alpha}} \varphi_{2}(x, 0)-\frac{\mu}{s^{\alpha}} \frac{\partial^{\alpha} \varphi_{2}(s, t)}{\partial x^{\alpha}} \\
& =\frac{E_{\alpha}\left(x^{\alpha}\right)}{s^{\alpha}}-\frac{\mu}{s^{\alpha}}\left(\frac{E_{\alpha}\left(x^{\alpha}\right)}{s^{\alpha}}-\frac{\mu E_{\alpha}\left(x^{\alpha}\right)}{s^{2 \alpha}}+\frac{\mu^{2} E_{\alpha}\left(x^{\alpha}\right)}{s^{3 \alpha}}\right) \\
& =\frac{E_{\alpha}\left(x^{\alpha}\right)}{s^{\alpha}}-\frac{\mu E_{\alpha}\left(x^{\alpha}\right)}{s^{2 \alpha}}+\frac{\mu^{2} E_{\alpha}\left(x^{\alpha}\right)}{s^{3 \alpha}}-\frac{\mu^{3} E_{\alpha}\left(x^{\alpha}\right)}{s^{4 \alpha}} .
\end{aligned}
$$

Applying (26) and (29) gives the fourth approximation

$$
\begin{aligned}
\widetilde{L}_{\alpha} & \left\{\varphi_{4}(x, t)\right\} \\
& =\widetilde{L}_{\alpha}\left\{\varphi_{3}(x, t)\right\}-\frac{1}{s^{\alpha}} \widetilde{L}_{\alpha}\left\{\frac{\partial^{\alpha} \varphi_{3}(x, t)}{\partial t^{\alpha}}+\mu \frac{\partial^{\alpha} \varphi_{3}(x, t)}{\partial x^{\alpha}}\right\} \\
& =\varphi_{3}(x, s)-\frac{1}{s^{\alpha}}\left\{s^{\alpha} \varphi_{3}(x, s)-\varphi_{3}(x, 0)+\mu \frac{\partial^{\alpha} \varphi_{3}(x, s)}{\partial x^{\alpha}}\right\} \\
& =\frac{1}{s^{\alpha}} \varphi_{3}(x, 0)-\frac{\mu}{s^{\alpha}} \frac{\partial^{\alpha} \varphi_{3}(s, t)}{\partial x^{\alpha}} \\
& =\frac{E_{\alpha}\left(x^{\alpha}\right)}{s^{\alpha}}-\frac{\mu}{s^{\alpha}}\left(\frac{E_{\alpha}\left(x^{\alpha}\right)}{s^{\alpha}}-\frac{\mu E_{\alpha}\left(x^{\alpha}\right)}{s^{2 \alpha}}\right. \\
& \left.+\frac{\mu^{2} E_{\alpha}\left(x^{\alpha}\right)}{s^{3 \alpha}}-\frac{\mu^{3} E_{\alpha}\left(x^{\alpha}\right)}{s^{4 \alpha}}\right)
\end{aligned}
$$

$$
\begin{aligned}
= & \frac{E_{\alpha}\left(x^{\alpha}\right)}{s^{\alpha}}-\frac{\mu E_{\alpha}\left(x^{\alpha}\right)}{s^{2 \alpha}}+\frac{\mu^{2} E_{\alpha}\left(x^{\alpha}\right)}{s^{3 \alpha}} \\
& -\frac{\mu^{3} E_{\alpha}\left(x^{\alpha}\right)}{s^{4 \alpha}}+\frac{\mu^{4} E_{\alpha}\left(x^{\alpha}\right)}{s^{4 \alpha}} .
\end{aligned}
$$

In view of (26) and (31), we give the fifth approximation

$$
\begin{aligned}
\widetilde{L}_{\alpha}\left\{\varphi_{5}(x, t)\right\} & \widetilde{L}_{\alpha}\left\{\varphi_{4}(x, t)\right\}-\frac{1}{s^{\alpha}} \widetilde{L}_{\alpha}\left\{\frac{\partial^{\alpha} \varphi_{4}(x, t)}{\partial t^{\alpha}}+\mu \frac{\partial^{\alpha} \varphi_{4}(x, t)}{\partial x^{\alpha}}\right\} \\
= & \varphi_{4}(x, s)-\frac{1}{s^{\alpha}}\left\{s^{\alpha} \varphi_{4}(x, s)-\varphi_{4}(x, 0)+\mu \frac{\partial^{\alpha} \varphi_{4}(x, s)}{\partial x^{\alpha}}\right\} \\
= & \frac{1}{s^{\alpha}} \varphi_{4}(x, 0)-\frac{\mu}{s^{\alpha}} \frac{\partial^{\alpha} \varphi_{4}(s, t)}{\partial x^{\alpha}} \\
= & \frac{E_{\alpha}\left(x^{\alpha}\right)}{s^{\alpha}}-\frac{\mu}{s^{\alpha}}\left(\frac{E_{\alpha}\left(x^{\alpha}\right)}{s^{\alpha}}-\frac{\mu E_{\alpha}\left(x^{\alpha}\right)}{s^{2 \alpha}}+\frac{\mu^{2} E_{\alpha}\left(x^{\alpha}\right)}{s^{3 \alpha}}\right. \\
= & \left.\frac{E_{\alpha}\left(x^{\alpha}\right)}{s^{\alpha}}-\frac{\mu E_{\alpha}\left(x^{\alpha}\right)}{s^{2 \alpha}}+\frac{\mu^{2} E_{\alpha}\left(x^{\alpha}\right)}{s^{3 \alpha}}-\frac{\mu^{4} E_{\alpha}\left(x^{\alpha}\right)}{s^{4 \alpha}}\right) \\
& +\frac{\mu^{4} E_{\alpha}\left(x^{\alpha}\right)}{s^{4 \alpha}}-\frac{\mu^{5} E_{\alpha}\left(x^{\alpha}\right)}{s^{5 \alpha}} .
\end{aligned}
$$

Therefore, we obtain

$$
\begin{aligned}
\widetilde{L}_{\alpha}\left\{\varphi_{n}(x, t)\right\} \\
\quad=\lim _{n \rightarrow \infty} \widetilde{L}_{\alpha}\left\{\frac{1}{\mu} \sum_{i=1}^{n} \frac{\mu^{(1+2 i)} E_{\alpha}\left(x^{\alpha}\right)}{s^{(1+2 i) \alpha}}-\frac{1}{\mu} \sum_{i=1}^{n} \frac{\mu^{2 i} E_{\alpha}\left(x^{\alpha}\right)}{s^{2 i \alpha}}\right\},
\end{aligned}
$$

which reduces to

$$
\begin{aligned}
& \varphi(x, t) \\
& =\lim _{n \rightarrow \infty}\left\{\frac{1}{\mu} \sum_{i=1}^{n} \frac{\mu^{(1+2 i)} E_{\alpha}\left(x^{\alpha}\right)}{s^{(1+2 i) \alpha}}-\frac{1}{\mu} \sum_{i=1}^{n} \frac{\mu^{2 i} E_{\alpha}\left(x^{\alpha}\right)}{s^{2 i \alpha}}\right\} \\
& =E_{\alpha}\left(x^{\alpha}\right) \lim _{n \rightarrow \infty}\left\{\frac{1}{\mu} \sum_{i=1}^{n} \frac{\mu^{(1+2 i)}}{s^{(1+2 i) \alpha}}-\frac{1}{\mu} \sum_{i=1}^{n} \frac{\mu^{2 i}}{s^{2 i \alpha}}\right\} \\
& =E_{\alpha}\left(x^{\alpha}\right)\left(\sum_{i=1}^{n} \frac{\mu^{2 i} t^{2 i \alpha}}{\Gamma(1+2 i \alpha)}-\sum_{i=1}^{n} \frac{\mu^{2 i-1} t^{(2 i-1) \alpha}}{\Gamma(1+(2 i-1) \alpha)}\right) \\
& =E_{\alpha}\left(x^{\alpha}\right)\left[\cos _{\alpha}\left(\mu t^{\alpha}\right)-\sin _{\alpha}\left(\mu t^{\alpha}\right)\right]
\end{aligned}
$$

and its graph is shown in Figure 1 with the parameters $\alpha=$ $\ln 2 / \ln 3$ and $\mu=2$. 


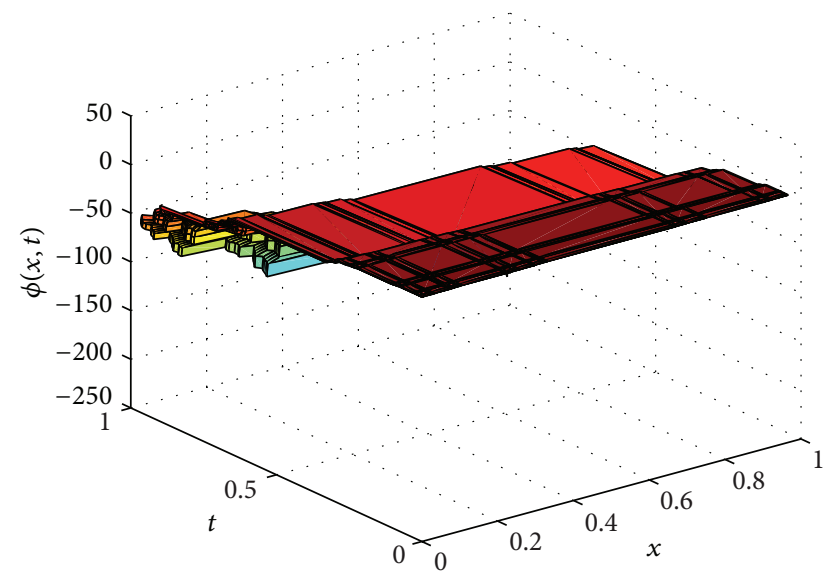

Figure 1: The graph of (1) with the parameters $\alpha=\ln 2 / \ln 3$ and $\mu=2$.

Example 2. The initial and boundary conditions for line partial differential equations arising in fractal vehicular traffic flow with the parameter $\mu=1$ are

$$
\begin{gathered}
\varphi(x, 0)=\sinh _{\alpha}\left(x^{\alpha}\right), \\
\varphi(0, t)=0 .
\end{gathered}
$$

From (21), we obtain

$$
\begin{aligned}
\widetilde{L}_{\alpha} & \left\{\varphi_{n+1}(x, t)\right\} \\
& =\widetilde{L}_{\alpha}\left\{\varphi_{n}(x, t)\right\}-\frac{1}{s^{\alpha}} \widetilde{L}_{\alpha}\left\{\frac{\partial^{\alpha} \varphi_{n}(x, t)}{\partial t^{\alpha}}+\frac{\partial^{\alpha} \varphi_{n}(x, t)}{\partial x^{\alpha}}\right\} \\
& =\varphi_{n}(x, s)-\frac{1}{s^{\alpha}}\left\{s^{\alpha} \varphi_{n}(x, s)-\varphi_{n}(x, 0)+\frac{\partial^{\alpha} \varphi_{n}(x, s)}{\partial x^{\alpha}}\right\} \\
& =\frac{1}{s^{\alpha}} \varphi_{n}(x, 0)-\frac{1}{s^{\alpha}} \frac{\partial^{\alpha} \varphi_{n}(s, t)}{\partial x^{\alpha}},
\end{aligned}
$$

where the initial value is

$$
\widetilde{L}_{\alpha}\left\{\varphi_{0}(x, t)\right\}=\varphi_{0}(x, s)=\widetilde{L}_{\alpha}\left\{\sinh _{\alpha}\left(x^{\alpha}\right)\right\}=\frac{\sinh _{\alpha}\left(x^{\alpha}\right)}{s^{\alpha}} .
$$

In view of (36) and (37), we get the first approximation

$$
\begin{aligned}
\widetilde{L}_{\alpha} & \left\{\varphi_{1}(x, t)\right\} \\
= & \widetilde{L}_{\alpha}\left\{\varphi_{0}(x, t)\right\}-\frac{1}{s^{\alpha}} \widetilde{L}_{\alpha}\left\{\frac{\partial^{\alpha} \varphi_{0}(x, t)}{\partial t^{\alpha}}+\frac{\partial^{\alpha} \varphi_{0}(x, t)}{\partial x^{\alpha}}\right\} \\
= & \varphi_{0}(x, s)-\frac{1}{s^{\alpha}}\left\{s^{\alpha} \varphi_{0}(x, s)-\varphi_{0}(x, 0)+\frac{\partial^{\alpha} \varphi_{0}(x, s)}{\partial x^{\alpha}}\right\} \\
= & \frac{1}{s^{\alpha}} \varphi_{0}(x, 0)-\frac{1}{s^{\alpha}} \frac{\partial^{\alpha} \varphi_{0}(s, t)}{\partial x^{\alpha}} \\
= & \frac{\sinh _{\alpha}\left(x^{\alpha}\right)}{s^{\alpha}}-\frac{\cosh _{\alpha}\left(x^{\alpha}\right)}{s^{\alpha}} .
\end{aligned}
$$

From (36) and (38), we obtain the second approximation as follows:

$$
\begin{aligned}
\widetilde{L}_{\alpha} & \left\{\varphi_{2}(x, t)\right\} \\
& =\widetilde{L}_{\alpha}\left\{\varphi_{1}(x, t)\right\}-\frac{1}{s^{\alpha}} \widetilde{L}_{\alpha}\left\{\frac{\partial^{\alpha} \varphi_{1}(x, t)}{\partial t^{\alpha}}+\frac{\partial^{\alpha} \varphi_{1}(x, t)}{\partial x^{\alpha}}\right\} \\
& =\varphi_{1}(x, s)-\frac{1}{s^{\alpha}}\left\{s^{\alpha} \varphi_{1}(x, s)-\varphi_{1}(x, 0)+\frac{\partial^{\alpha} \varphi_{1}(x, s)}{\partial x^{\alpha}}\right\} \\
& =\frac{1}{s^{\alpha}} \varphi_{1}(x, 0)-\frac{1}{s^{\alpha}} \frac{\partial^{\alpha} \varphi_{1}(s, t)}{\partial x^{\alpha}} \\
& =\frac{\sinh _{\alpha}\left(x^{\alpha}\right)}{s^{\alpha}}-\frac{1}{s^{\alpha}}\left(\frac{\cosh _{\alpha}\left(x^{\alpha}\right)}{s^{\alpha}}+\frac{\sinh _{\alpha}\left(x^{\alpha}\right)}{s^{\alpha}}\right) \\
& =\frac{\sinh _{\alpha}\left(x^{\alpha}\right)}{s^{\alpha}}-\frac{\cosh _{\alpha}\left(x^{\alpha}\right)}{s^{2 \alpha}}-\frac{\sinh _{\alpha}\left(x^{\alpha}\right)}{s^{2 \alpha}} .
\end{aligned}
$$

Using (36) and (39), we have the third approximation

$$
\begin{aligned}
\widetilde{L}_{\alpha}\left\{\varphi_{3}(x, t)\right\} \\
=\widetilde{L}_{\alpha}\left\{\varphi_{2}(x, t)\right\}-\frac{1}{s^{\alpha}} \widetilde{L}_{\alpha}\left\{\frac{\partial^{\alpha} \varphi_{2}(x, t)}{\partial t^{\alpha}}+\frac{\partial^{\alpha} \varphi_{2}(x, t)}{\partial x^{\alpha}}\right\} \\
=\varphi_{2}(x, s)-\frac{1}{s^{\alpha}}\left\{s^{\alpha} \varphi_{2}(x, s)-\varphi_{2}(x, 0)+\frac{\partial^{\alpha} \varphi_{2}(x, s)}{\partial x^{\alpha}}\right\} \\
=\frac{1}{s^{\alpha}} \varphi_{2}(x, 0)-\frac{1}{s^{\alpha}} \frac{\partial^{\alpha} \varphi_{2}(s, t)}{\partial x^{\alpha}} \\
=\frac{\sinh _{\alpha}\left(x^{\alpha}\right)}{s^{\alpha}}-\frac{1}{s^{\alpha}} \frac{\partial^{\alpha}}{\partial x^{\alpha}}\left(\frac{\sinh _{\alpha}\left(x^{\alpha}\right)}{s^{\alpha}}-\frac{\cosh _{\alpha}\left(x^{\alpha}\right)}{s^{2 \alpha}}\right. \\
\left.=\frac{\sinh _{\alpha}\left(x^{\alpha}\right)}{s^{\alpha}}\right) \\
\left.s^{3 \alpha}\right) \\
s^{2 \alpha}-\frac{\cosh _{\alpha}\left(x^{\alpha}\right)}{s^{3 \alpha}}+\frac{\cosh _{\alpha}\left(x^{\alpha}\right)}{s^{4 \alpha}} .
\end{aligned}
$$

Making use of (36) and (40), we have the fourth approximation

$$
\begin{aligned}
\widetilde{L}_{\alpha} & \left\{\varphi_{4}(x, t)\right\} \\
& =\widetilde{L}_{\alpha}\left\{\varphi_{3}(x, t)\right\}-\frac{1}{s^{\alpha}} \widetilde{L}_{\alpha}\left\{\frac{\partial^{\alpha} \varphi_{3}(x, t)}{\partial t^{\alpha}}+\frac{\partial^{\alpha} \varphi_{3}(x, t)}{\partial x^{\alpha}}\right\} \\
& =\varphi_{3}(x, s)-\frac{1}{s^{\alpha}}\left\{s^{\alpha} \varphi_{3}(x, s)-\varphi_{3}(x, 0)+\frac{\partial^{\alpha} \varphi_{3}(x, s)}{\partial x^{\alpha}}\right\} \\
& =\frac{1}{s^{\alpha}} \varphi_{3}(x, 0)-\frac{1}{s^{\alpha}} \frac{\partial^{\alpha} \varphi_{3}(s, t)}{\partial x^{\alpha}}
\end{aligned}
$$




$$
\begin{aligned}
= & \frac{\sinh _{\alpha}\left(x^{\alpha}\right)}{s^{\alpha}}-\frac{1}{s^{\alpha}} \frac{\partial^{\alpha}}{\partial x^{\alpha}}\left(\frac{\sinh _{\alpha}\left(x^{\alpha}\right)}{s^{\alpha}}-\frac{\cosh _{\alpha}\left(x^{\alpha}\right)}{s^{2 \alpha}}\right. \\
& \left.\quad-\frac{\sinh _{\alpha}\left(x^{\alpha}\right)}{s^{3 \alpha}}+\frac{\cosh _{\alpha}\left(x^{\alpha}\right)}{s^{4 \alpha}}\right) \\
= & \frac{\sinh _{\alpha}\left(x^{\alpha}\right)}{s^{\alpha}}-\frac{\cosh _{\alpha}\left(x^{\alpha}\right)}{s^{2 \alpha}}-\frac{\sinh _{\alpha}\left(x^{\alpha}\right)}{s^{3 \alpha}} \\
& +\frac{\cosh _{\alpha}\left(x^{\alpha}\right)}{s^{4 \alpha}}+\frac{\sinh _{\alpha}\left(x^{\alpha}\right)}{s^{5 \alpha}} .
\end{aligned}
$$

From (36) and (41), we get the fifth approximation as follows:

$$
\begin{aligned}
\widetilde{L}_{\alpha}\left\{\varphi_{5}(x, t)\right\} & \widetilde{L}_{\alpha}\left\{\varphi_{4}(x, t)\right\}-\frac{1}{s^{\alpha}} \widetilde{L}_{\alpha}\left\{\frac{\partial^{\alpha} \varphi_{4}(x, t)}{\partial t^{\alpha}}+\frac{\partial^{\alpha} \varphi_{4}(x, t)}{\partial x^{\alpha}}\right\} \\
= & \varphi_{4}(x, s)-\frac{1}{s^{\alpha}}\left\{s^{\alpha} \varphi_{4}(x, s)-\varphi_{4}(x, 0)+\frac{\partial^{\alpha} \varphi_{4}(x, s)}{\partial x^{\alpha}}\right\} \\
= & \frac{1}{s^{\alpha}} \varphi_{4}(x, 0)-\frac{1}{s^{\alpha}} \frac{\partial^{\alpha} \varphi_{4}(s, t)}{\partial x^{\alpha}} \\
= & \frac{\sinh h_{\alpha}\left(x^{\alpha}\right)}{s^{\alpha}} \\
& -\frac{1}{s^{\alpha}} \frac{\partial^{\alpha}}{\partial x^{\alpha}}\left(\frac{\sinh _{\alpha}\left(x^{\alpha}\right)}{s^{\alpha}}-\frac{\cosh _{\alpha}\left(x^{\alpha}\right)}{s^{2 \alpha}}-\frac{\sinh _{\alpha}\left(x^{\alpha}\right)}{s^{3 \alpha}}\right. \\
& \left.+\frac{\cosh _{\alpha}\left(x^{\alpha}\right)}{s^{4 \alpha}}+\frac{\sinh _{\alpha}\left(x^{\alpha}\right)}{s^{5 \alpha}}\right) \\
& +\frac{\sinh _{\alpha}\left(x^{\alpha}\right)}{s^{\alpha}}-\frac{\cosh _{\alpha}\left(x^{\alpha}\right)}{s^{2 \alpha}}-\frac{\sinh _{\alpha}\left(x^{\alpha}\right)}{s^{3 \alpha}}+\frac{\cosh _{\alpha}\left(x^{\alpha}\right)}{s^{4 \alpha}} \\
s^{5 \alpha} & \frac{\cosh _{\alpha}\left(x^{\alpha}\right)}{s^{6 \alpha}} \cdot
\end{aligned}
$$

Thus, we obtain the local fractional series as follows:

$$
\begin{aligned}
\widetilde{L}_{\alpha} & \left\{\varphi_{n}(x, t)\right\} \\
& =\lim _{n \rightarrow \infty} \widetilde{L}_{\alpha}\left\{\sum_{i=0}^{n}(-1)^{i} \frac{\sinh _{\alpha}\left(x^{\alpha}\right)}{s^{(2 i+1) \alpha}}-\sum_{i=0}^{n}(-1)^{i} \frac{\cosh _{\alpha}\left(x^{\alpha}\right)}{s^{2 i \alpha}}\right\},
\end{aligned}
$$

which yields

$$
\begin{gathered}
\varphi(x, t) \\
=\lim _{n \rightarrow \infty}\left\{\sum_{i=0}^{n}(-1)^{i} \frac{\sinh _{\alpha}\left(x^{\alpha}\right) t^{2 i \alpha}}{\Gamma(1+2 i \alpha)}\right. \\
\left.-\sum_{i=0}^{n}(-1)^{i} \frac{\cosh _{\alpha}\left(x^{\alpha}\right) t^{(2 i+1) \alpha}}{\Gamma(1+(2 i+1) \alpha)}\right\} \\
=\sinh _{\alpha}\left(x^{\alpha}\right) \cos _{\alpha}\left(t^{\alpha}\right)-\cosh _{\alpha}\left(x^{\alpha}\right) \sin _{\alpha}\left(t^{\alpha}\right),
\end{gathered}
$$

and its graph is shown in Figure 2.

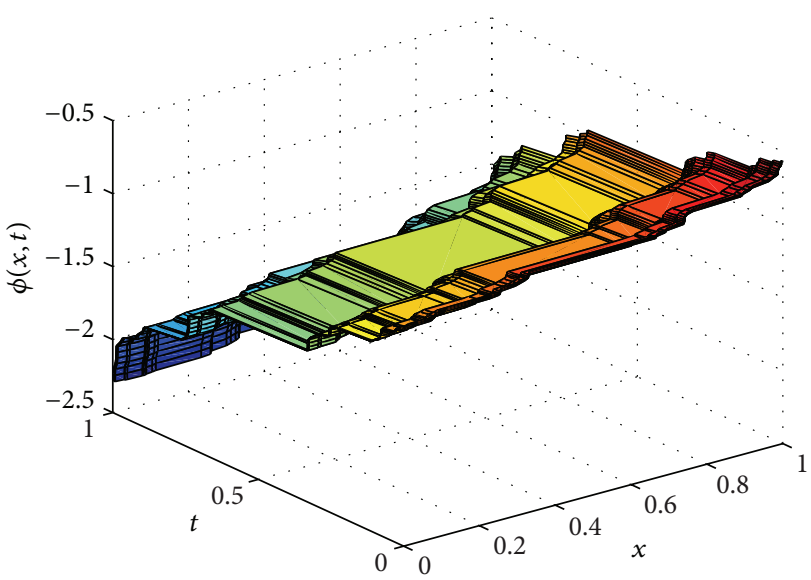

FIGURE 2: The graph of (1) with the parameters $\alpha=\ln 2 / \ln 3$ and $\mu=1$.

\section{Conclusions}

In this paper, the boundary value problems for line partial differential equations arising in fractal vehicular traffic flow were solved by using the local fractional Laplace variational iteration method, which is the coupling method of local fractional variational iteration method (a generalization of variational iteration method based upon the local fractional calculus) and Laplace transform (a generalization of Fourier transform based upon the local fractional calculus). The nondifferentiable approximate solutions were obtained and their graphs were also shown.

\section{Conflict of Interests}

The authors declare that they have no conflict of interests regarding the publication of this paper.

\section{References}

[1] V. E. Tarasov, "Wave equation for fractal solid string," Modern Physics Letters B, vol. 19, no. 15, pp. 721-728, 2005.

[2] S. Momani and Z. Odibat, "Analytical approach to linear fractional partial differential equations arising in fluid mechanics," Physics Letters A: General, Atomic and Solid State Physics, vol. 355, no. 4-5, pp. 271-279, 2006.

[3] S. Momani and Z. Odibat, "Analytical solution of a timefractional Navier-Stokes equation by Adomian decomposition method," Applied Mathematics and Computation, vol. 177, no. 2, pp. 488-494, 2006.

[4] Y. Z. Povstenko, "Fractional heat conduction equation and associated thermal stress," Journal of Thermal Stresses, vol. 28, no. 1, pp. 83-102, 2005.

[5] L. Vázquez, J. J. Trujillo, and M. P. Velasco, "Fractional heat equation and the second law of thermodynamics," Fractional Calculus and Applied Analysis, vol. 14, no. 3, pp. 334-342, 2011.

[6] E. Lutz, "Fractional transport equations for Lévy stable processes," Physical Review Letters, vol. 86, no. 11, pp. 2208-2211, 2001. 
[7] A. Kadem, Y. Luchko, and D. Baleanu, "Spectral method for solution of the fractional transport equation," Reports on Mathematical Physics, vol. 66, no. 1, pp. 103-115, 2010.

[8] N. Laskin, "Fractional Schrödinger equation," Physical Review E. Statistical, Nonlinear, and Soft Matter Physics, vol. 66, no. 5, Article ID 056108, 7 pages, 2002.

[9] S. I. Muslih, O. P. Agrawal, and D. Baleanu, "A fractional Schrödinger equation and its solution," International Journal of Theoretical Physics, vol. 49, no. 8, pp. 1746-1752, 2010.

[10] D. Baleanu, K. Diethelm, E. Scalas, and J. J. Trujillo, Fractional Calculus Models and Numerical Methods, vol. 3 of Complexity, Nonlinearity and Chaos, World Scientific, Boston, Mass, USA, 2012.

[11] H. Jafari and S. Seifi, "Homotopy analysis method for solving linear and nonlinear fractional diffusion-wave equation," Communications in Nonlinear Science and Numerical Simulation, vol. 14, no. 5, pp. 2006-2012, 2009.

[12] H. Jafari, H. Tajadodi, N. Kadkhoda, and D. Baleanu, "Fractional subequation method for Cahn-Hilliard and KleinGordon equations," Abstract and Applied Analysis, vol. 2013, Article ID 587179, 5 pages, 2013.

[13] J. Hristov, "Heat-balance integral to fractional (half-time) heat diffusion sub-model," Thermal Science, vol. 14, no. 2, pp. 291-316, 2010.

[14] J. Hristov, "Transient flow of a generalized second grade fluid due to a constant surface shear stress: an approximate integralbalance solution," International Review of Chemical Engineering, vol. 3, no. 6, pp. 802-809, 2011.

[15] A. H. Bhrawy and M. A. Alghamdi, "A shifted Jacobi-GaussLobatto collocation method for solving nonlinear fractional Langevin equation involving two fractional orders in different intervals," Boundary Value Problems, vol. 2012, article 62, 2012.

[16] A. H. Bhrawy and M. M. Al-Shomrani, "A shifted Legendre spectral method for fractional-order multi-point boundary value problems," Advances in Difference Equations, vol. 2012, article 8, 2012.

[17] A. H. Bhrawy and D. Baleanu, "A spectral Legendre-GaussLobatto collocation method for a space-fractional advection diffusion equations with variable coefficients," Reports on Mathematical Physics, vol. 72, no. 2, pp. 219-233, 2013.

[18] A. Atangana and D. Baleanu, "Numerical solution of a kind of fractional parabolic equations via two difference schemes," Abstract and Applied Analysis, vol. 2013, Article ID 828764, 8 pages, 2013.

[19] X.-J. Yang, Advanced Local Fractional Calculus and Its Applications, World Science, New York, NY, USA, 2012.

[20] X.-J. Yang, H. M. Srivastava, J.-H. He, and D. Baleanu, "Cantortype cylindrical-coordinate method for differential equations with local fractional derivatives," Physics Letters A, vol. 377, no. 28-30, pp. 1696-1700, 2013.

[21] Y. Zhao, D. Baleanu, C. Cattani, D.-F. Cheng, and X.-J. Yang, "Maxwell's equations on Cantor sets: a local fractional approach," Advances in High Energy Physics, vol. 2013, Article ID 686371, 6 pages, 2013.

[22] X.-J. Yang and D. Baleanu, "Fractal heat conduction problem solved by local fractional variation iteration method," Thermal Science, vol. 17, no. 2, pp. 625-628, 2013.

[23] C. F. Liu, S. S. Kong, and S. J. Yuan, "Reconstructive schemes for variational iteration method within Yang-Laplace transform with application to fractal heat conduction problem," Thermal Science, vol. 17, no. 3, pp. 715-721, 2013.
[24] X. J. Yang, D. Baleanu, and J. T. Machado, "Application of the local fractional Fourier series to fractal signals," in Discontinuity and Complexity in Nonlinear Physical Systems, pp. 63-89, Springer, 2014.

[25] K. Liu, R.-J. Hu, C. Cattani, G.-N. Xie, X.-J. Yang, and Y. Zhao, "Local fractional $Z$-transforms with applications to signals on cantor sets," Abstract and Applied Analysis, vol. 2014, Article ID 638648, 6 pages, 2014.

[26] G. Yi, "Local fractional Z transform in fractal space," Advances in Digital Multimedia, vol. 1, no. 2, pp. 96-102, 2012.

[27] X.-J. Yang, D. Baleanu, H. M. Srivastava, and J. A. Tenreiro Machado, "On local fractional continuous wavelet transform," Abstract and Applied Analysis, vol. 2013, Article ID 725416, 5 pages, 2013.

[28] X.-J. Yang, D. Baleanu, and J. A. Tenreiro Machado, "Systems of Navier-Stokes equations on Cantor sets," Mathematical Problems in Engineering, vol. 2013, Article ID 769724, 8 pages, 2013.

[29] X.-J. Yang, D. Baleanu, and J. A. Tenreiro Machado, "Mathematical aspects of Heisenberg uncertainty principle within local fractional Fourier analysis," Boundary Value Problems, vol. 2013, article 131, 2013.

[30] C.-Y. Long, Y. Zhao, and H. Jafari, "Mathematical models arising in the fractal forest gap via local fractional calculus," Abstract and Applied Analysis, vol. 2014, Article ID 782393, 6 pages, 2014.

[31] L.-F. Wang, X.-J. Yang, D. Baleanu, C. Cattani, and Y. Zhao, "Fractal dynamical model of vehicular traffic flow within the local fractional conservation laws," Abstract and Applied Analysis, vol. 2014, Article ID 635760, 5 pages, 2014.

[32] J.-H. He and F.-J. Liu, "Local fractional variational iteration method for fractal heat transfer in silk cocoon hierarchy," Nonlinear Science Letters A, vol. 4, no. 1, pp. 15-20, 2013.

[33] A. M. Yang, J. Li, H. M. Srivastava, G. N. Xie, and X. J. Yang, "The local fractional Laplace variational iteration method for solving linear partial differential equations with local fractional derivatives," Discrete Dynamics in Nature and Society, vol. 2014, Article ID 365981, 2014.

[34] Y. Z. Zhang, A. M. Yang, and Y. Long, "Initial boundary value problem for fractal heat equation in the semi-infinite region by Yang-Laplace transform," Thermal Science, 2013.

[35] C.-G. Zhao, A.-M. Yang, H. Jafari, and A. Haghbin, “The YangLaplace transform for solving the IVPs with local fractional derivative," Abstract and Applied Analysis, vol. 2014, Article ID 386459, 5 pages, 2014. 


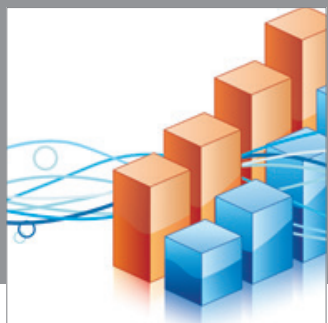

Advances in

Operations Research

mansans

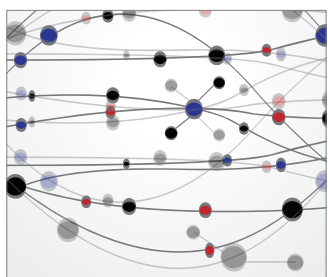

The Scientific World Journal
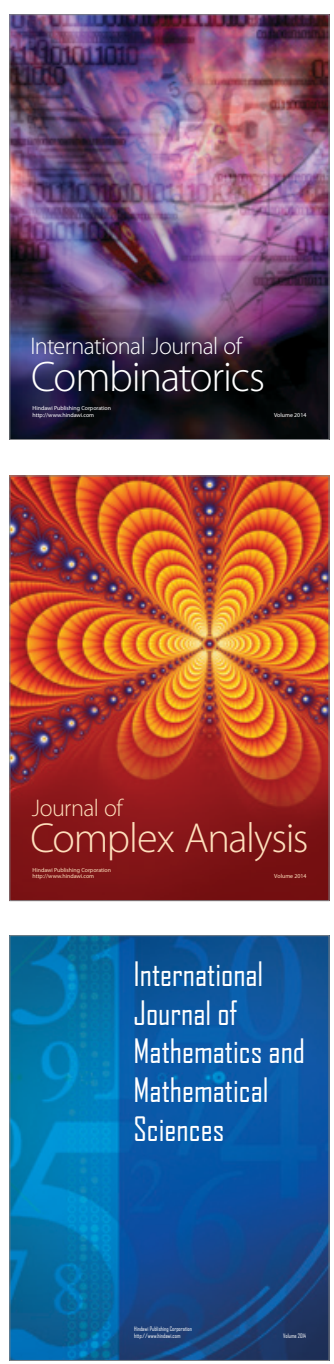
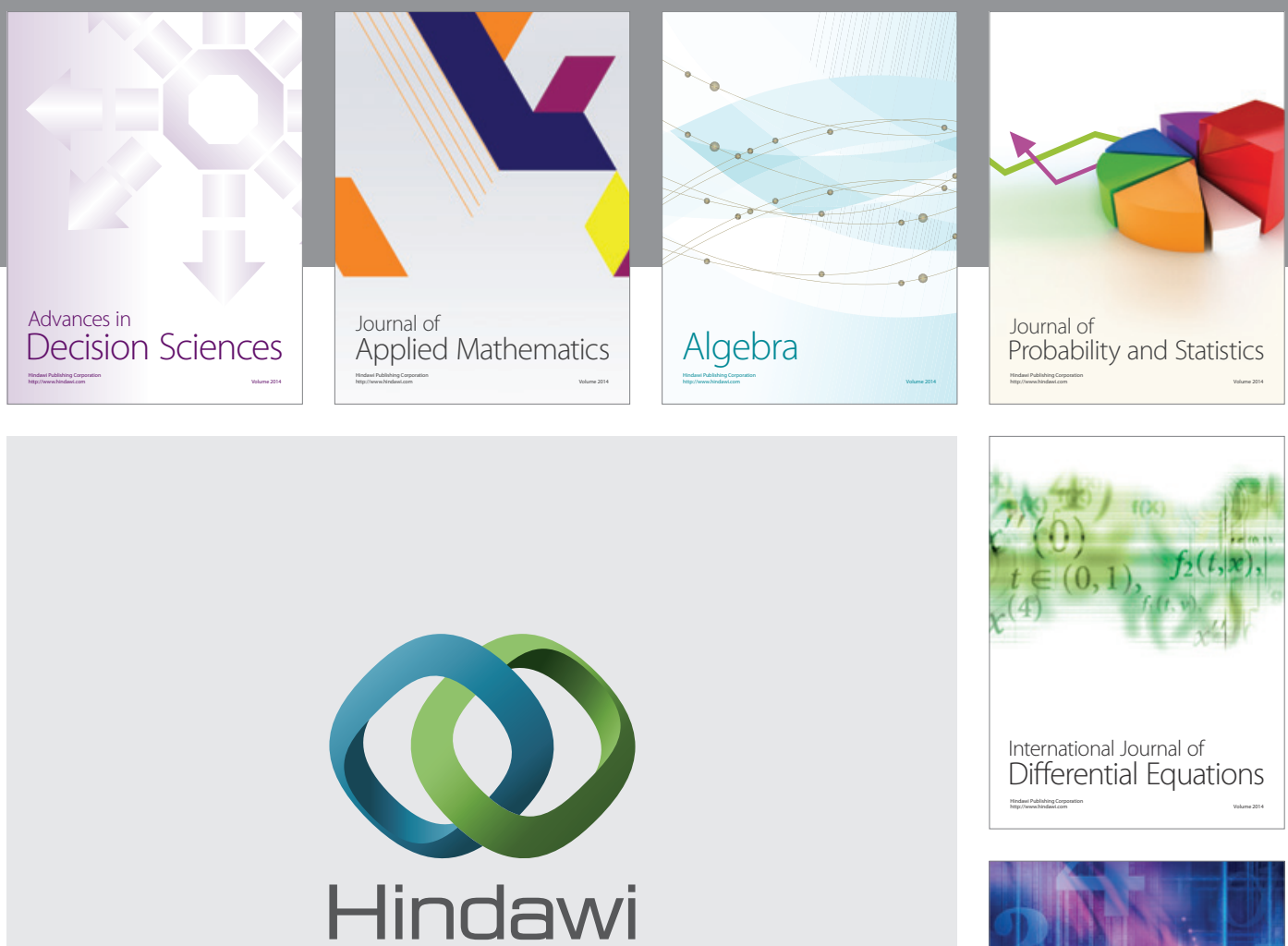

Submit your manuscripts at http://www.hindawi.com
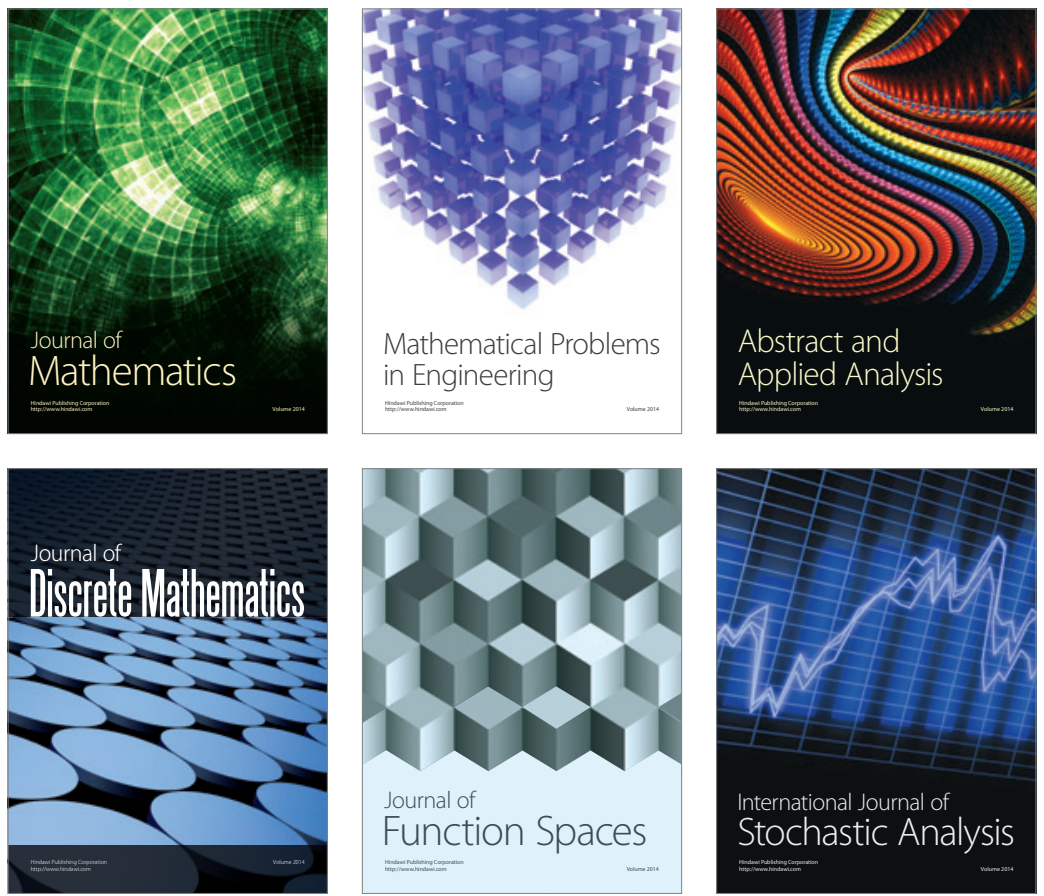

Journal of

Function Spaces

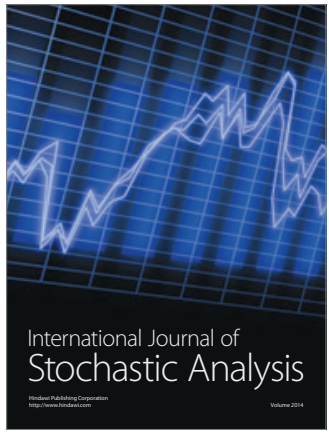

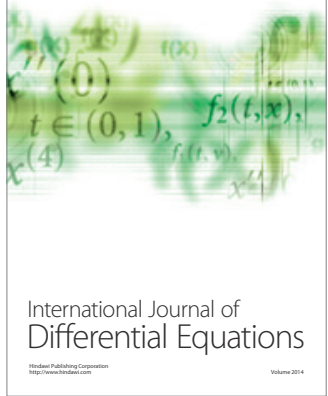
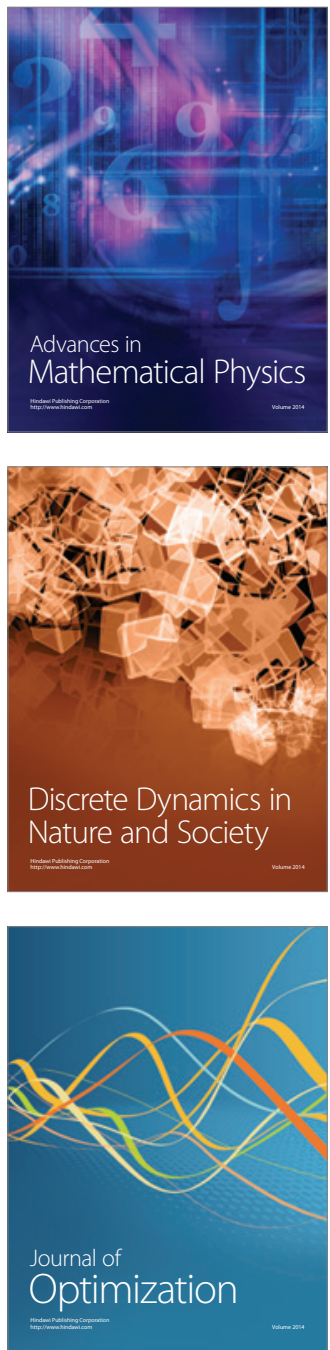